\title{
AN IMPROVED ENERGY EFFICIENT CHAIN-BASED ROUTING PROTOCOL USING ACO
}

\author{
Aya A. Hussein ${ }^{1}$, Rajaaaldeen A. Khalid ${ }^{2}$ \\ 1,2 College of Information Engineering, AL-Nahrain University, Baghdad, Iraq \\ aya.ayad.it@gmail.com ${ }^{1}$, rajaaldeen@ coie-nahrain.edu.iq ${ }^{2}$ \\ Received:26/9/2019, Accepted:10/10/2019
}

\begin{abstract}
As new ideas and perspectives continue to be shared by various authors, many different aspects of WSN technology have been generated according to the pivotal role of WSN for many fields in our lives. This technology contains different components, the most basic one is the sensor nodes that communicate with each other and deliver the data. Routing protocols make the sensors' communication and delivering of data thoughtfully planned to be more reliable. Routing protocols are a wide field given the importance of their presence in the network to route the data to the best path or shortest path as much as possible among sensor nodes. This paper focus on cluster-based routing protocols that have hierarchal architecture, this class of protocols has gone through a lot of developments and improvements because of the great benefits that offer to WSN. Part of these improvements that applied to IEEPB protocol (Improved Energy- Efficient PEGASIS- Based protocol) which is present in this paper is enhancing the protocol work mechanism using the Ant colony optimization technique (ACO) to conserve wasted energy and thus increase the network lifetime. Therefore, this paper proposes a SIEEPB- ACO (Single IEEPB - ACO) protocol that is an improved protocol over the IEEPB protocol using ACO. The protocol is simulated using the Matlab R2018b simulator which provides clear and effective simulation results, especially for energy saving. Therefore, due to the simulation results, SIEEPB-ACO provides a noticeable improvement compared to the PEGASIS and IEEPB protocols.
\end{abstract}

keywords: WSN, Cluster- based routing protocols, IEEPB protocol, Ant Colony Optimization (ACO), Energy efficient, Network lifetime.

\section{INTRODUCTION}

Despite the successful WSN effectiveness on several fields in technology, there still some constraint that Restricted workflow of routing protocols in WSN or effect on the quality of service offered by any WSN such as energy consumption due to the limited power supply of each sensor. Therefore, it was necessary to enhance the working mechanism of WSN to overcome energy- wasting and other problems related to this kind of network. As a result, one of the effective ways is to enhance routing protocols work mechanism to make the communication between sensor nodes more reliable without wasting energy or lose information. Hierarchal routing protocols is a cluster- based routing protocol which is one of three categories that specified based on the network architecture of WSN as showing below in Fig. 1 [1], [2], [3], [4]. This paper will focus on a chain- based routing protocol especially, Pegasis protocol that is already an improved protocol over LEACH protocol. However, PEGASIS protocol has been improved by many authors in different ways that will be mention in a quick review in the next related work section. The main aim of this paper is to choose one of the chain- based routing protocols (IEEPB) to improve it to (SIEEPB- ACO): (Single Improved Energy- Efficient PEGASIS- Based protocol- Ant Colony Optimization) by adding an ant colony optimization system to enhance the behavior of IEEPB and compare the performance of the protocol after and before using the ACO technique. Therefore, the paper will be organized as follow. Section 1, the introduction. While Section 2, explains the related work. Section 3 deals with the proposed work theoretical 
background. And section 4, describes the proposed work design flow. Finally, Section 5 presents the system simulation and results for the proposed protocol.Finally, section 6 concludes the paper and suggest some beneficial future work.

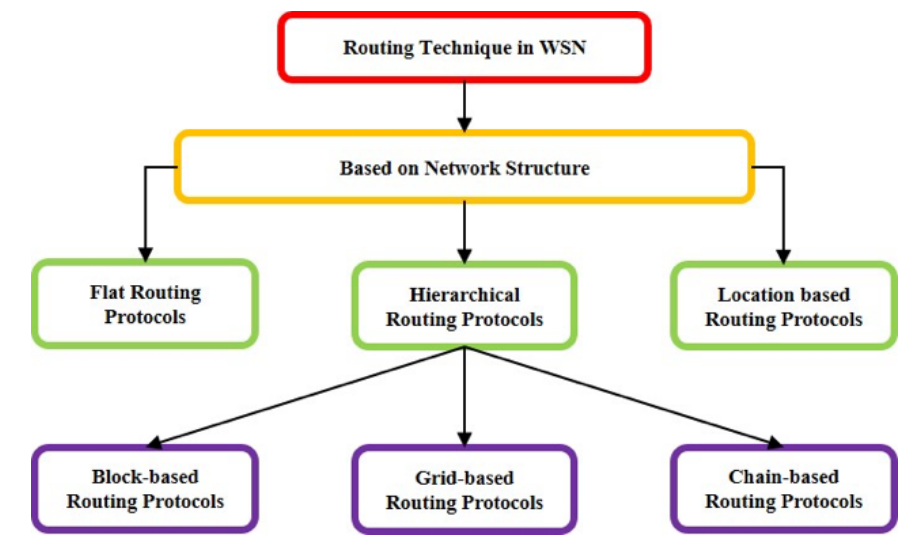

Figure 1: Routing technique based on network structure in WSN

\section{Literature Review}

WSN is a wild field of research and studies, so many authors go deeply in this field to discover its huge benefits that satisfy human needs. However, in this section, you will find a preview for different author's ideas but for the same goal, the goal of reaching the best routing protocol to get the best results. The author in [5] presents a wide clear description of different routing protocols structures such as flat, hierarchal, and QoS network routing protocols. Were flat network routing protocols are explained based on their hybrid, reactive and proactive routing protocols while hierarchal network routing protocols are explained based on their network architecture: chain, grid, and location-based hierarchal routing protocols. In addition, the author takes several protocols for the above- mentioned network routing protocols classification as an example and show work mechanism conception, attributes, merits, and demerits amply. The protocol that was the beginning of openness for hierarchal routing protocols is LEACH protocol (Low-Energy Adaptive Clustering Hierarchy) that proposed by the author of reference [6] LEACH protocol divide the data among the sensor nodes with a hierarchical structure in WSN, it is known as a block-based routing protocol. The main goal of this protocol is to prolong network lifetime according to the clustering concept. In spite of that, this protocol has its own drawbacks due to its scalability because of the thesis of this protocol States that (any node can send and receive the data from any other node in the network). This thesis can cause selecting inappropriate locations to distribute the cluster heads. Therefore a new protocol has been proposed in [7] that is an improved protocol over LEACH protocol to overcome the effected drawbacks caused by previous protocol. PEGASIS Protocol (power-efficient gathering in sensor information systems) known as chain based routing protocol, its technique is to Forms chains between sensor nodes that guarantee delivering data in more reliable way by collecting data from each node and aggregate it in accumulative way to reach the final node that is leader node which collect all the data and send it to the base station. However, this protocol works efficiently for large scale area otherwise it is not preferable small scale areas. In reference [8] the author proposes an improved protocol over the PEGASIS protocol called EEPB protocol (Energy-Efficient 
PEGASIS- Based protocol) to solve the problem of Long Links (LL Problem). EEPB solves and recovers several problems over PEGASIS but still has some drawbacks that been solved in the IEEPB protocol. In reference [9] the author proposes IEEPB protocol (Improved Energy- Efficient PEGASIS- Based protocol) to overcome the deficiencies of EEPB by using several ways that will be described deeply in the next few pages in section3 (Proposed Work Background). The author in [10] proposed PRACO (a predication mode routing algorithm based on ACO), this algorithm examines the load factor for each path or branch in heuristic factor and generate a new pheromone updating rule. As a result, this algorithm will have the ability to know previously and adaptively the situation and condition of each node in WSN this, in turn, achieve efficient usages of energy for data gathering routing structure in wireless sensor networks. In [11] MIEEPB protocol (Mobile sink improved energy- efficient PEGASIS- based routing protocol) is proposed by the author to modify the IEEPB protocol by adding the mobility to the sink node. Moving sink node is Moves around its path for a certain sojourn time and location to collect all the data efficiently. In [12] the author uses Ant colony optimization to obtain an optimal chain in a chain based routing protocol for WSNs to propose three scenarios: PEG- ACO, Horizontal PEG- ACO clustering, and vertical PEGACO clustering. All these scenarios are compared with the core protocol PEGASIS, so The aim of this work is to prolong the network lifetime due to the number of alive nodes and consume energy in an efficient manner according to changing the sensor node distribution in WSN area with the use of ACO. The author show results for several performance metrics, which show enhancing for network lifetime and energy conservation and will be mention with more detail in simulation and result section in this paper. In ref. [13] the author presents an Improvement of Multi-Chain PEGASIS Using Relative Distance where it can help to allow BS (base station) to generate the table of location information and forms the multitopology without the need for GPS. In addition, transmission concept works according to the aggregation of the changed data only so as to minimize amount of data transmission.

\section{Proposed Work Background}

\section{A. Proposed Routing Protocol:}

As been described previously about the genesis of IEEPB protocol in section2 (Literature Review) according to (LEACHPEGASIS- EEPB - IEEPB - proposed protocol) which is shown in the next Fig. 2 In this section, we will present in details a clear wide description about the main goal of EEPB protocol and the drawbacks that carriage the researchers to propose an improved protocol over EEPB protocol called IEEPB . this, in turn, will lead us to give the reasons behind the idea of enhancing IEEPB protocol by using ACO and propose SIEEPB- ACO protocol.

1) Routing Protocols : EEPB protocol is a single chain based routing protocol, it is improved protocol over PEGASIS protocol by the author of ref. [8] to overcome the problem of long links caused by PEGASIS protocol. The main techniques that been used in EEPB to get rid of LL problem are [13], [14]:

- While the single- chain is construct a threshold is arranged on this chain to minimize the construction of LL.

- The leader node is selected according to both the effective terms :

- residual energy of nodes.

- the distance between the node and the BS(Base Station).

https://ijict.edu.iq 
- Set the other next reselections leader nodes times according to the remaining number of nodes.

EEPB protocol had a weakly effectiveness to solve the problems of PEGASIS protocols.so in spite of the fact that it's been solving some issues in PEGASIS it still had some deficiencies [14], [15]:

- The threshold that adopts on the chain was uncertainty and this, in turn, did not solve the problem of LL.

- In the stage of selecting the leader node sometimes EEPB ignores part of the suitable amount of remaining energy of nodes and the distance between them and BS.

Therefore, the author in ref. [9] propose an improved protocol over the EEPB protocol called IEEPB protocol to overcome these effective drawbacks [14],[15]. IEEPB specification was based on:

- In the beginning, the protocol compares the distance between two neighbored nodes for double-time to ensure finding the shortest path between these two nodes.

- To select the leader node this protocol also considering the same two effective terms in EEPB that are residual energy of the nodes and distance between the node and the BS but the difference in IEEPB that this protocol distributes different weights coefficients to these two terms. So, based on these weights, the node that has the lowest weight will be selected as leader node.

- The previous specification can be expressed in the following equations that are applied only on IEEPB protocol :

To calculate the distance:

$$
D_{b s}=d_{T o B S} / d_{\text {ave }}
$$

Where:

$D_{b s}$ : Distance between the Base station and sensor node.

$d_{T o B S}$ : Distance from the sensor node to BS.

$d_{a v e}:$ Average distance. To calculate the residual energy and the normalized energy :

$$
E_{p}=E_{\text {init }} / E_{i}
$$

Where:

$E_{p}:$ Energy portion.

$E_{\text {init }}:$ Initial energy of node i.

$E_{i}$ : Residual energy of node $\mathrm{i}$ for round $\mathrm{n}$.

to find the weight of a certain node:

$$
\begin{gathered}
W_{i}=w_{1} E_{p}+w_{2} D_{b s} \\
w_{1}+w_{2}=1
\end{gathered}
$$

Where:

$W_{i}$ : combined weight for node $\mathrm{i}$.

$w_{1}, w_{2}$ :The coefficient of weight factors. As a result, the lowest node weight will be selected to be the leader node 
in this round [9], [11]. In spite of these changes and improvements that applied only on the IEEPB protocol. IEEPB still needed to more enhancement because of the drawbacks that raised such as:

- There is a high load on the chain because of the protocol still suffering from LL problems.

- High delays ratio.

So, due to these deficiencies, this paper will present an improvement over the IEEPB protocol with different calculation rules for energy calculations and leader node selection criteria and forming shorter chains using Ant Colony Algorithm (ACA) that will present in the next few pages instead of the greedy algorithm that been used to be applied for the previous protocols.

\section{2) Ant Colony Algorithm (ACA):}

Ant Colony Optimization algorithm is an optimization system inspired by nature according to the real ant behavior to get the food to the colony. Ants searching in wild fields around the colony to find any destination that has food. So, if an ant finds a food source, it returns to the colony, leaving behind a trail of pheromones. As a result, the rest of the ants that are directly behind the first and will follow the pheromones trail of the previous ant. When this group of ant takes the food and come back to the colony it strengthens the pheromone trails to help the rest of ants to find the source food smoothly and to keep the trail of pheromone a way from evaporations and over the time the pheromone trail will be considered as the best path (optimal in global) for all ants but then when food source is disappearing for any reason this trail pheromone will be evaporated and disappeared gradually since not all the ant will go through it and they will find another food source by using their ability of discovering. In the world of simulation, these ant is called agents where they can communicate and cooperate with each other through pheromone trails. Each node in the whole network will be visited by each ant randomly. The following equations will be used in proposed protocol SIEEPB-ACO so for the first equation is present the transition probability, $\mathrm{P}$, of which next node $\mathrm{q}$ will be chosen by the ant $\mathrm{k}$ [16], [17], [18]:

$$
\operatorname{Prob}(p, q)=\left\{\begin{array}{cl}
\frac{\left[\tau(p, q)^{\alpha}\right] \cdot[\eta(p, q)]^{\beta}}{\sum_{q \varepsilon R_{q}\left[\tau_{i j}(t)\right]^{\alpha} \cdot\left[\eta_{i j}\right]^{\beta}}} & \text { if } q \notin M^{k} \\
0 & \text { otherwise }
\end{array}\right.
$$

Where:

$\operatorname{Prob}(p, q):$ are the pheromone created by the ant.

$\eta(p, q)$ : is the heuristic function for energy and distance.

$R_{q}:$ is the recipient nodes.

$M^{k}:$ is the list of earlier visited nodes for node $\mathrm{p}$.

$\alpha$ : is the exponential weight of the pheromone.

$\beta:$ is the heuristic exponential weight of the pheromone.

Where also, to get an optimal solution based on the transition probabilities which is given in Eq. 5. it is recommended to use the Roulette Wheel Selection method [16], [17], [18]. However, to control the amounts of pheromone and find 
a new optimal tour the evaporation mechanism of pheromone should be applied by using the following equation:

$$
\tau_{p q}=(1-p) \tau_{p q}
$$

Where:

$0<q \geq 1$ :is the evaporation rate.

$\tau_{p q}:$ is the pheromone level or amounts between node $\mathrm{p}$ and node $\mathrm{q}$.

The following equation shows the keeping of pheromone on a trail when an ant come back to the nest.

$$
\Delta \tau^{k}=Q\left(\frac{1}{L^{k}}\right)
$$

Where

$\tau^{k}:$ is the amount of kept pheromone.

$L^{k}:$ is the distance between ant $\mathrm{k}$ in terms of visited hops and $\mathrm{Q}$ is the weighting coefficient.

Hence, the trail pheromone level will change based on the following equation:

$$
\tau=\tau+\Delta \tau^{k}
$$

After all of these steps that are obvious in Fig. 3 are finished and the ants end their journey, the best route or the best path will be found. so, the steps of this process continue to be repeated to the point of reaching the globally optimal path. Moreover, to gain a global optimal chain of shortest distance, ACO is advised to use because its proven superiority compared to other optimization algorithms such as Simulated Annealing and Genetic Algorithm, in solving the Travelling Salesman Problem where shortest path connecting multiple points need to be found ( see Fig. 4 ) [19] [20].

3) Proposed routing protocol: In this paper, the proposed protocol is based on the IEEPB protocol with its threshold and all the conditions that are already an improvement over the PEGASIS protocol as mention previously but with adding a swarm intelligence algorithm.

- proposed protocol benefits:

The proposed protocol (SIEEPB-ACO) improves IEEPB protocol (single chain /single cluster head) by using an artificial intelligence algorithm: ant colony optimization algorithm to:

- Eliminate the maximization of the distance between nodes compared with IEEPB or PEGASIS protocol which based on the greedy algorithm.

- A globally optimal chain is formatted because of multiple ACO rounds that calculated to choose the best cost function.

- Minimize the effect of long links problems by finding the shortest path based on ACO.

- Minimize the energy consumption of the network.

- Increase and prolong the network lifetime based on the node death of the network.

- proposed protocol construction: 


\section{- Formation of a Chain:}

In the beginning, achieve reliable communication between nodes by the construct of neighbored sensor nodes using ACO with it eq. 5, 6, 7,and 8 to find the shortest path based on the distance between each node and form the chain according to the best route that found (see Fig. 5).

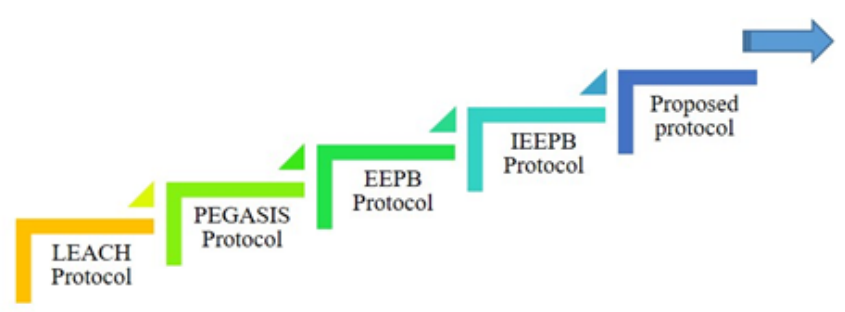

Figure 2: Hierarchical routing protocols improvements
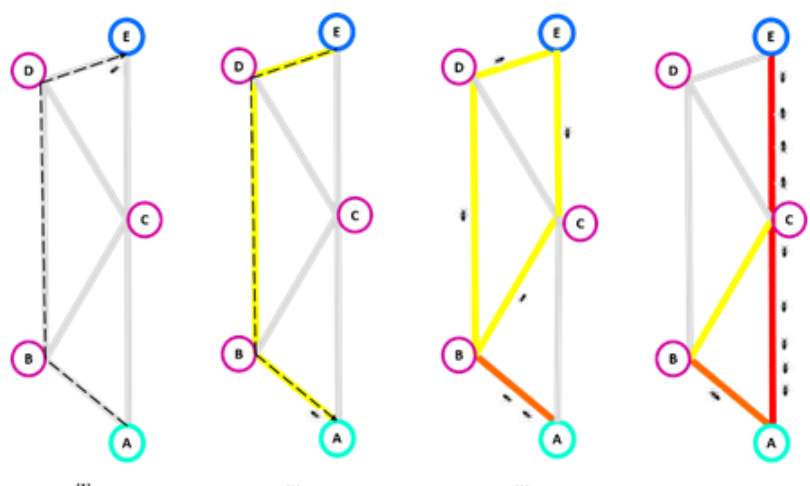

Figure 3: Basic ACO behavior

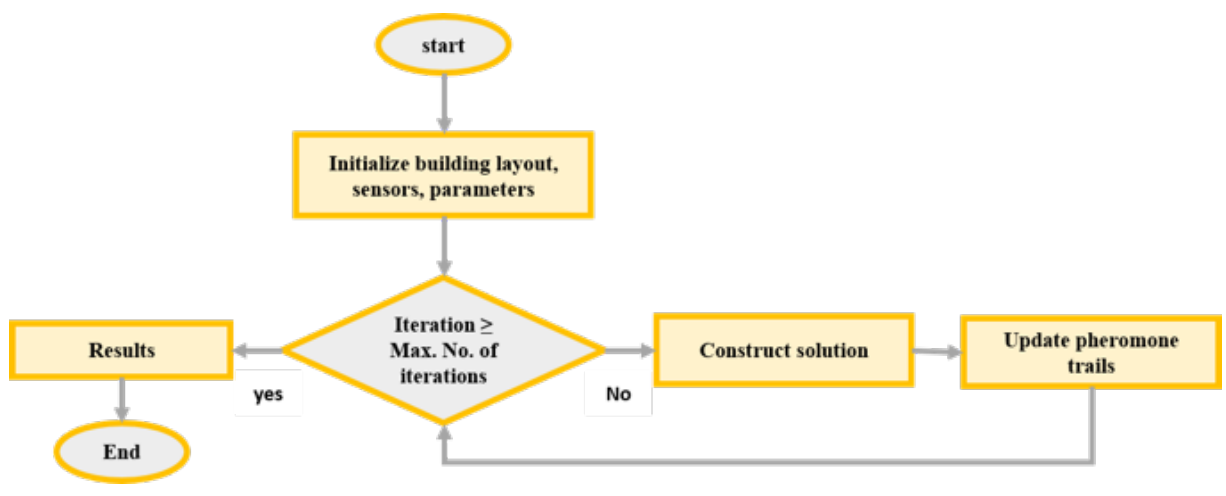

Figure 4: Flowchart of ACO 


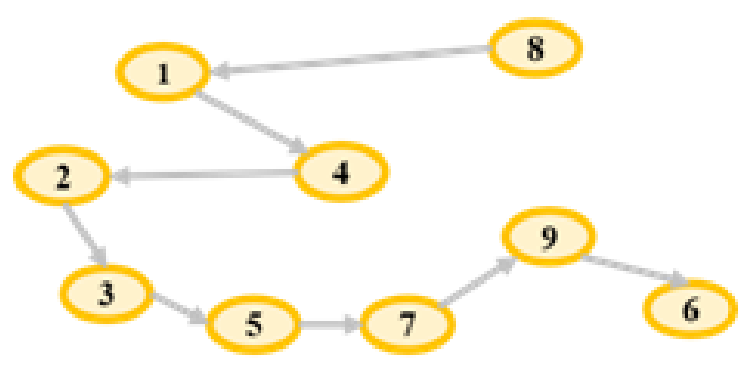

Figure 5: Chain formation

- Chain leader selection.

The selection of the best node as a leader node in the SIEEPB- ACO protocol will be according to the weight rule that is obvious in eq. 9 only, it calculates each node weight and chooses the highest node weight as the primary leader node. Chain chooses the chain leader according to the weight Q dedicated to each node. Each node divides its residual energy with its distance from the base station to computes its weight Q. The weights of all nodes in the chain will be compared by the network. Then, the network computes the node that has the highest weight and chooses it as a primary chain leader of the chain. Then the secondary leader node is selected based on comparing the distance between the node and its parent node $\left(D_{p}\right)$ with the distance between the same node and the sink node $\left(D_{s}\right)$. If $D_{s}<D_{p}$ then the node will act as a secondary leader node and transfer the data to the sink node instead of sending it to parent node.

$$
Q_{i}=\frac{E_{i}}{D_{i}}
$$

Where:

$Q_{i}$ : calculated weight for node i.

$E_{i}$ : Residual energy of sensor node $\mathrm{i}$.

$D_{i}$ : The distance between sensor node $\mathrm{i}$ and sink.

- Data transmission: (Collect, gather, and forward data).

All The data from each node and it is neighbor will be merge and forward this gathered data to the next node as an accumulative way until reaching the leader node or we can call it cluster head $(\mathrm{CH})$. Thus, the leader node will be responsible for sending this data to the base station. Moreover, in each round there is a new chain is formatted based on ACO and a new leader node is selected based on the previous chain leader selection concept due to the continues process of nodes death. In a certain round, the leader will initiate a control token passing approach to obtain the gathered data from the further node to the nearest one and forward it to the base station. In Fig. 6 the leader node is $\mathrm{n} 3$ so the first token will be pass from n0 through $\mathrm{n} 1$ then $\mathrm{n} 2$ and reach the leader node $\mathrm{n} 3$ then the second token will be pass from $\mathrm{n} 6$ through $\mathrm{n} 5$ then $\mathrm{n} 4$ and also reach the leader node $\mathrm{n} 3$. All 
the data from the two directors will be gathered in $\mathrm{n} 3$ then forward to the base station.

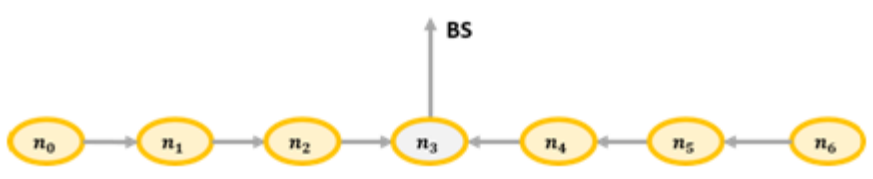

Figure 6: Control token passing approach in the chain-based routing protocols

- network model: This paper presents two protocols to be compared:

a) Original PEGASIS based on the greedy algorithm.

b) Original single IEEPB based on the greedy algorithm.

c) Proposed SIEEPB-ACO based on the ACO algorithm.

d) For reliability of results, All the previous mention protocols are simulated in the same network parameters (see

Table I), the same Computer and simulation tool capability (see table II), and same locations of sensor nodes (see table III).

e) Simulated for two scenarios :

- With initial energy $E_{0}=0.5 \mathrm{~J}$.

- With initial energy $E_{0}=1 J$.

f) In WSN areas size $(100 m \times 100 m)$ with the number of nodes (100). In addition, a fixed sink node location is considered in the center of the network size $(50 \mathrm{~m} \times 50 \mathrm{~m})$. Considering that the effect of signal interference in a wireless channel is ignored. thus, to calculate energy consumption in data transmission that consumed by each sensor, a first-order radio model is employed as shown in the next equation [21]:

$$
\begin{gathered}
E_{t x}(k, d)=k \times E_{\text {elec }}+k \times E_{\text {amp }} \times d^{2}, \text { when }\left(d<d_{0}\right) \\
E_{t x}(k, d)=k \times E_{\text {elec }}+k \times E_{\text {amp }} \times d^{4}, \text { when }\left(d \geq d_{0}\right) \\
E_{r x}(k)=k \times E_{\text {elec }}
\end{gathered}
$$

Based on first order radio model in Fig. 7, Where:

$E_{\text {elec }}$ : The energy consumed by the radio to run the transmitter or receiver circuitry, (equal to $50 \mathrm{~nJ} / \mathrm{bit}$ ).

$E_{a m p}$ : The required energy for transmitter amplifier in free space, (equal to $100 \mathrm{pJ} / \mathrm{bit} / \mathrm{m}^{2}$ ).

$E_{m p}:$ The required energy for the transmitter amplifier in the multipath model.

$E_{D A}$ : The energy consumed by Transmitter circuitry to aggregate the data received by the child nodes, (equal to $50 n J / b i t)$.

$\mathrm{k}$ : Number of bits.

$d^{2}$ : Represent power loss in free space with $\mathrm{d} 2$ and with $\mathrm{d} 4$ power loss.

$d^{4}$ : Represent power loss in multipath fading. 
$d_{0}:$ Represent threshold.

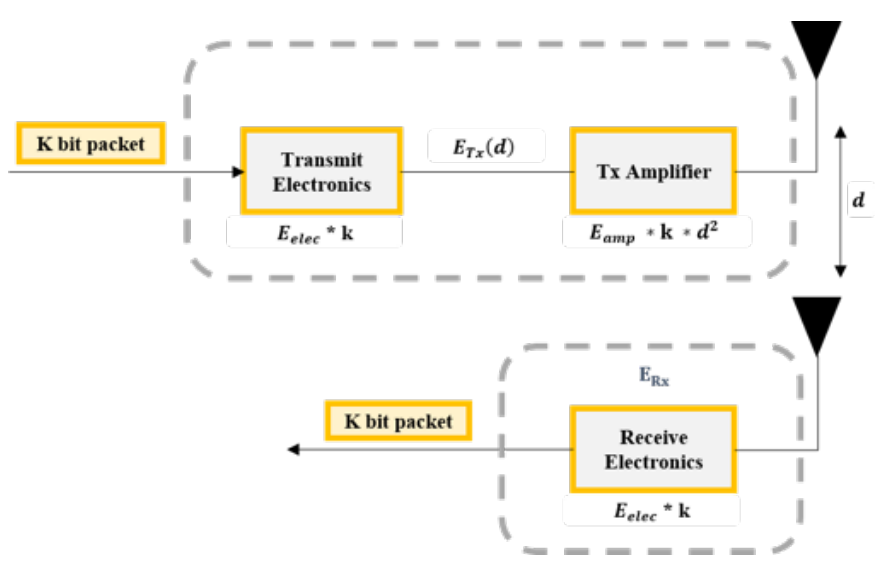

Figure 7: First-order radio model

To calculate the distance:

$$
d_{i}=\sqrt{\left(x_{s}-x_{i}\right)^{2}+\left(y_{s}-y_{i}\right)^{2}}
$$

Where:

$d_{i}$ : Distance between the sink node and the sensor node.

$x_{s}, y_{s}$ : is the location of the sink node.

$x_{i}, y_{i}:$ is the location of $i$ node.

To calculate the residual energy per round :

$$
E_{R}=\sum_{i=1}^{n} E_{i}
$$

Where:

$E_{R}$ : Total residual energy for each round.

$\mathrm{n}$ : Number of nodes.

$E_{i}$ : Residual energy for node i.

To calculate normalized energy per round :

$$
E_{n}=\frac{\sum_{i}^{n} E i}{n * E_{o}}
$$

Where:

$E_{N}$ : Normalized energy for each round.

$E_{o}$ : Initial energy of node i. 


\section{Simulations And Results}

The proposed work has been simulated using Matlab simulator to examine and investigate enhanced single IEEPB using ACO respecting to its capability to expanding network lifetime by knowing the behavior of alive nodes and node death rate that will lead us to know important indicators to measure the performance of different algorithms such as network lifetime, number of alive nodes, residual energy of network, normalized energy of the network, and percentage of dead nodes. These indicators have been measured for a network size of $100 \mathrm{~m} \times 100 \mathrm{~m}$ with the sink node at the center of the area $(50,50)$, other common simulation parameters used are given in Table I. In addition, Fig. 8 shows the process steps to introduce this enhanced protocol and used for our simulations. The proposed protocol has been tested and compared with existing PEGASIS protocol and single IEEPB based greedy algorithm. For the reliability of results, the same parameters and networks were used for all routing methods. As it is obvious in the last two Fig. 9 for the first scenario, where $E_{0}=0.5 J$ : (a) IEEPB protocol, present the chain formation that was not optimal and many unneeded spaces are lifted behind between some nodes and this will lead high energy consumption without taking advantage of it but instead it causes damage for the network so as a result, it will minimize the network lifetime. On the other hand (b) SIEEPB- ACO protocol can show how the enhancement effecting on the shape of the chain on the total number of nodes and how the long and unneeded spaces are minimized. And for this (b) present an efficient improvement that will be clear in next Fig. 10 and Fig. 11 for the first scenario $E_{0}=0.5 J$ shows the number of alive nodes in PEGASIS, IEEPB, and proposed protocol with the enhancement that occurred by the SIEEPB- ACO.

TABLE I

NetWork PaRAmeters

\begin{tabular}{|c|c|}
\hline Parameter & Value \\
\hline Network Size & $100 \mathrm{~m} \times 100 \mathrm{~m}$ \\
\hline Number of nodes & 100 \\
\hline Initial energy & $0.5 J, 1 J$ \\
\hline Packet Size & 2000 \\
\hline Sink Location & $50 \mathrm{~m} \times 50 \mathrm{~m}$ \\
\hline Eelec & $50 \mathrm{~nJ} /$ bits \\
\hline Emp & $0.0013 \mathrm{pJ} /$ bit $^{\prime} \mathrm{m}^{4}$ \\
\hline Efs & $10 \mathrm{pJ} /$ bit $/ \mathrm{m}^{2}$ \\
\hline EDA & $5 n J /$ bit $/ \mathrm{message}^{4}$ \\
\hline Number of Rounds & 4000,8000 \\
\hline Threshold energy & $0.75,1.5$ \\
\hline Threshold energy & 50 \\
\hline Number of iteration & 700 \\
\hline$\rho$ & 0.5 \\
\hline$\alpha, \beta$ & 1 \\
\hline
\end{tabular}

TABLE II

COMPUTER AND SimUlation TOOL CAPABILITY

\begin{tabular}{|c|c|}
\hline Parameter & Value \\
\hline Computer processor & Core i7 \\
\hline processor frequency & $2.70 \mathrm{GHz}$ \\
\hline RAM & $12.0 \mathrm{~GB}$ \\
\hline Operating system & 64bit (windows 10) \\
\hline Simulation tool & Matlab R2018b \\
\hline
\end{tabular}




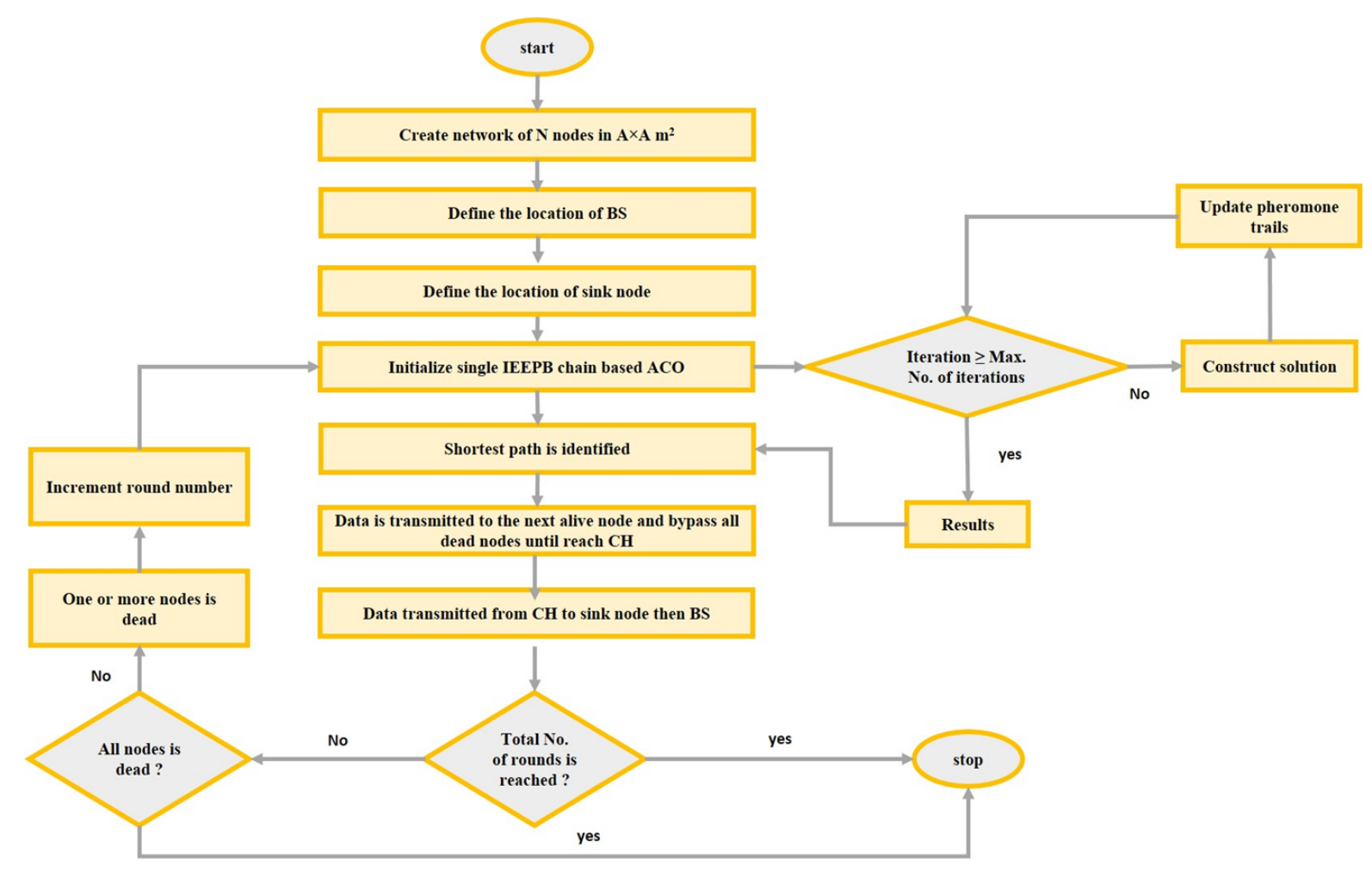

Figure 8: Flowchart of the proposed protocol

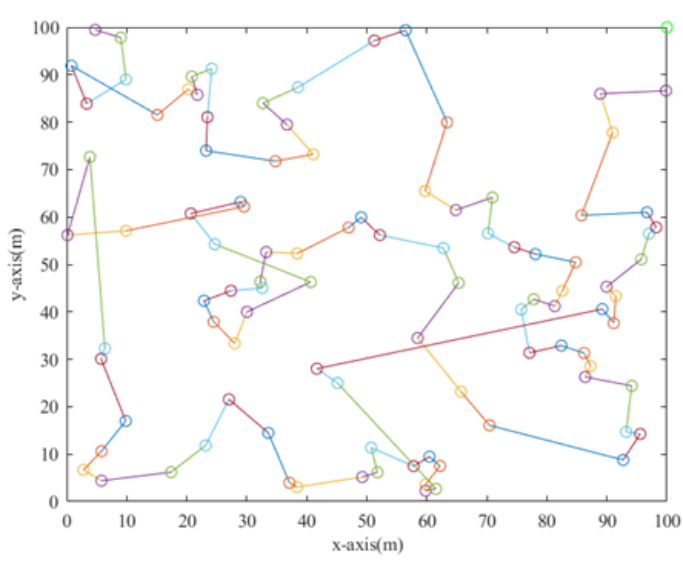

(a)

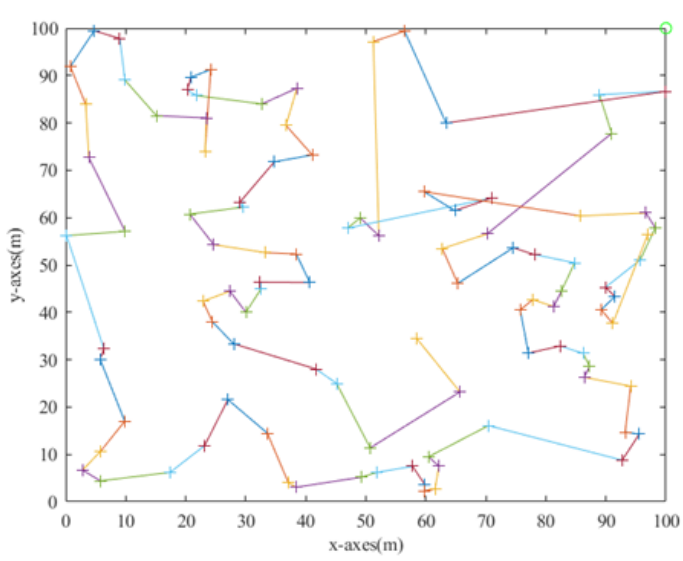

(b)

Figure 9: WSN area for: (a) IEEPB protocol (original), (b) SIEEPB- ACO protocol (proposed) 
TABLE III

FiXed SENSOR NODES LOCATIONS

\begin{tabular}{|c|c|c|c|c|c|c|c|c|c|c|c|}
\hline No. & X.d & Y.d & No. & X.d & Y.d & No. & X.d & Y.d & No. & X.d & Y.d \\
\hline 1 & 23.22548 & 73.98322 & 26 & 89.94264 & 45.29461 & 51 & 51.77235 & 6.182471 & 76 & 96.99652 & 56.48832 \\
\hline 2 & 88.89923 & 85.98076 & 27 & 5.802618 & 10.62694 & 52 & 23.13672 & 11.8486 & 77 & 21.76899 & 85.77633 \\
\hline 3 & 59.70608 & 65.47521 & 28 & 99.84253 & 86.63274 & 53 & 9.878004 & 89.02755 & 78 & 86.19993 & 31.33022 \\
\hline 4 & 91.5014 & 43.31831 & 29 & 61.52058 & 2.694448 & 54 & 3.338908 & 83.9035 & 79 & 32.69842 & 84.00134 \\
\hline 5 & 28.97608 & 63.18823 & 30 & 32.25226 & 46.37762 & 55 & 50.72667 & 11.37164 & 80 & 49.25328 & 5.190248 \\
\hline 6 & 29.54218 & 62.20288 & 31 & 9.901723 & 57.09881 & 56 & 49.04457 & 59.94417 & 81 & 77.80184 & 42.66724 \\
\hline 7 & 4.753419 & 99.46099 & 32 & 32.58751 & 45.04923 & 57 & 9.021613 & 97.8224 & 82 & 27.99619 & 33.3508 \\
\hline 8 & 20.67641 & 60.7364 & 33 & 57.78438 & 7.484431 & 58 & 65.30188 & 46.11321 & 83 & 36.694 & 79.47924 \\
\hline 9 & 34.76303 & 71.77371 & 34 & 5.734267 & 30.09564 & 59 & 86.37581 & 26.28291 & 84 & 3.869079 & 72.66693 \\
\hline 10 & 2.79933 & 6.684176 & 35 & 52.17212 & 56.18802 & 60 & 82.39629 & 32.89753 & 85 & 87.26845 & 28.58148 \\
\hline 11 & 92.70641 & 8.777176 & 36 & 24.15548 & 91.27202 & 61 & 94.13005 & 24.41081 & 86 & 65.68493 & 23.18866 \\
\hline 12 & 33.23986 & 52.61809 & 37 & 82.57343 & 44.45459 & 62 & 95.71102 & 51.0825 & 87 & 62.19539 & 7.512377 \\
\hline 13 & 24.66421 & 54.28995 & 38 & 98.20626 & 57.82676 & 63 & 56.45799 & 99.37161 & 88 & 96.67559 & 60.997 \\
\hline 14 & 78.08679 & 52.18824 & 39 & 23.44235 & 81.05904 & 64 & 77.09839 & 31.38019 & 89 & 38.37152 & 3.056977 \\
\hline 15 & 93.19499 & 14.7112 & 40 & 45.12742 & 24.99692 & 65 & 5.787262 & 4.407379 & 90 & 85.75656 & 60.35432 \\
\hline 16 & 41.67657 & 28.02929 & 41 & 95.54375 & 14.26503 & 66 & 81.2944 & 41.20733 & 91 & 84.78404 & 50.46162 \\
\hline 17 & 59.81003 & 3.647486 & 42 & 51.25634 & 97.19251 & 67 & 38.41632 & 52.31265 & 92 & 0.791084 & 91.90732 \\
\hline 18 & 6.36865 & 32.28825 & 43 & 64.83206 & 61.4671 & 68 & 89.2149 & 40.59206 & 93 & 41.07048 & 73.23657 \\
\hline 19 & 9.837853 & 17.00446 & 44 & 46.96504 & 57.77807 & 69 & 60.43889 & 9.480356 & 94 & 15.11053 & 81.52333 \\
\hline 20 & 37.11638 & 3.976162 & 45 & 91.13132 & 37.62203 & 70 & 33.59813 & 14.48418 & 95 & 90.96025 & 77.79425 \\
\hline 21 & 70.9241 & 64.13414 & 46 & 22.87648 & 42.35249 & 71 & 24.48805 & 37.89717 & 96 & 75.71231 & 40.51975 \\
\hline 22 & 17.40609 & 6.215194 & 47 & 27.35962 & 44.45658 & 72 & 27.03196 & 21.55993 & 97 & 70.19011 & 56.54609 \\
\hline 23 & 40.66594 & 46.30629 & 48 & 62.7515 & 53.46413 & 73 & 63.37311 & 79.97156 & 98 & 58.47364 & 34.49146 \\
\hline 24 & 20.26743 & 86.95499 & 49 & 38.54422 & 87.34501 & 74 & 20.8514 & 89.62562 & 99 & 70.42332 & 16.08637 \\
\hline 25 & 59.79404 & 2.301354 & 50 & 30.03487 & 40.00297 & 75 & 74.57095 & 53.63858 & 100 & 0.151393 & 56.20009 \\
\hline
\end{tabular}

Simulation result for scenario 1 , where $E_{0}=0.5 \mathrm{~J}$ :

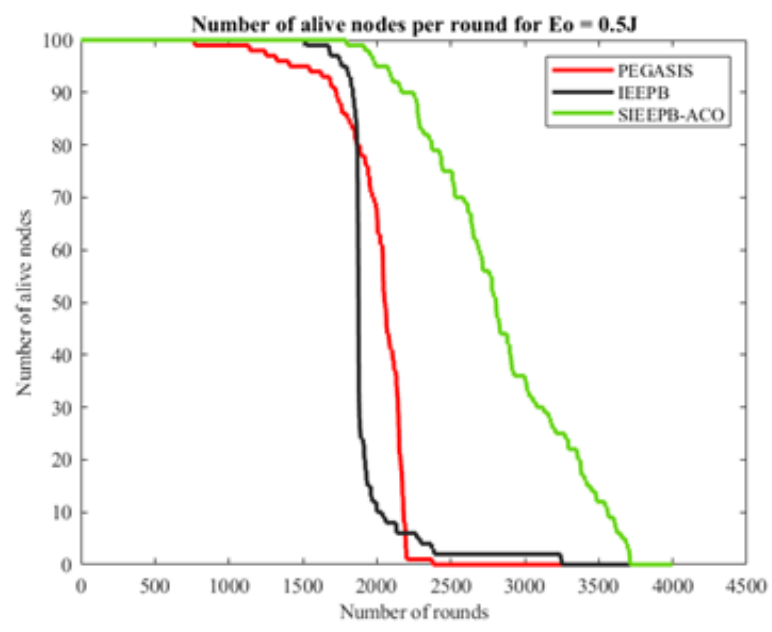

Figure 10: Number of alive nodes per round 


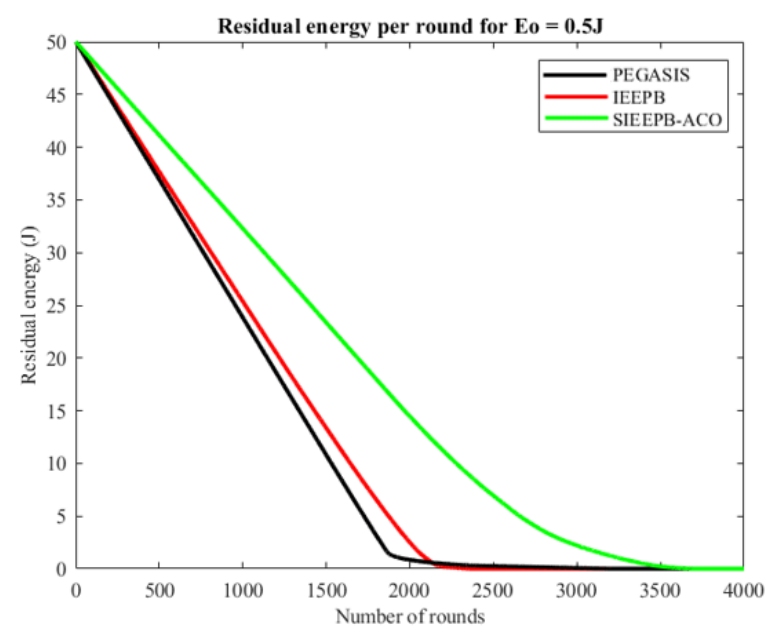

Figure 11: Residual energy per round

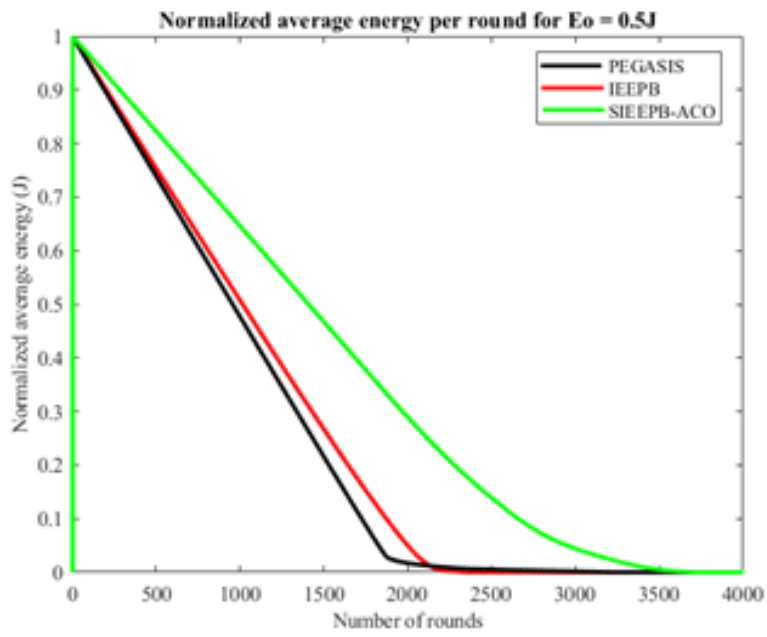

Figure 12: Normalized average energy per round

TABLE IV

NETWORK LIFETIME COMPARISION FOR $E_{o}=0.5 \mathrm{~J}$

\begin{tabular}{|c|c|c|c|}
\hline \multirow{2}{*}{ Nodes Death Rate } & \multicolumn{3}{|c|}{ Number of Rounds } \\
\cline { 2 - 4 } & PEGASIS & IEEPB & SIEEPB- ACO \\
\hline $1 \%$ & 770 & 1522 & 1798 \\
\hline $10 \%$ & 1722 & 1840 & 2166 \\
\hline $30 \%$ & 1969 & 1874 & 2529 \\
\hline $50 \%$ & 2052 & 1878 & 2806 \\
\hline $70 \%$ & 2143 & 1881 & 3078 \\
\hline $100 \%$ & 2377 & 3251 & 3713 \\
\hline
\end{tabular}




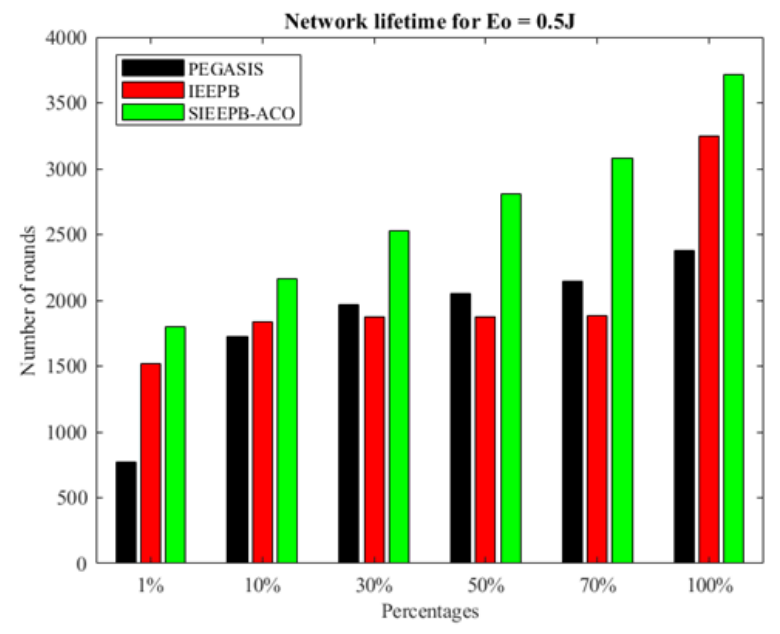

Figure 13: Comparison of network lifetime

For the first scenario, when $E_{0}=0.5 \mathrm{~J}$. The number of rounds (see table III) when sensor nodes start to die at $1 \%$. For example, the number 770 and 1522 in the first row (PEGASIS and IEEPB) means that the first dead node has been found after averagely 770 and 1522 rounds when the original PEGASIS and it is improvement IEEPB protocol applied in the WSN, at same time for the proposed protocol (SIEEPB- ACO) the number of rounds where nodes start to die was bigger than the previous ones and this means that the lifetime of the WSN is extended in SIEEPB-ACO as shown in Fig. 12 and Fig. 13 Same as when sensor nodes die at $10 \%$ and all other percentages. Thus, to be clearer you can notice the prolonging of network lifetime when $(1 \%, 10 \%, 30 \%, 50 \%$, and $100 \%)$ respectively of sensor nodes in the network have died. So, the results of the enhancement show the average life span in:

- $1 \%$ for SIEEPB- ACO is longer than the PEGASIS protocol by $133 \%$.

- $1 \%$ for SIEEPB- ACO is longer than IEEPB protocol by $18 \%$.

- $10 \%$ for SIEEPB- ACO is longer than the PEGASIS protocol by $26 \%$.

- $10 \%$ for SIEEPB- ACO is longer than IEEPB protocol by $18 \%$.

- $30 \%$ for SIEEPB- ACO is longer than the PEGASIS protocol by $28 \%$.

- $30 \%$ for SIEEPB- ACO is longer than IEEPB protocol by $35 \%$.

- $50 \%$ for SIEEPB- ACO is longer than the PEGASIS protocol by $37 \%$.

- $50 \%$ for SIEEPB- ACO is longer than IEEPB protocol by $50 \%$.

- $70 \%$ for SIEEPB- ACO is longer than the PEGASIS protocol by $44 \%$.

- $70 \%$ for SIEEPB- ACO is longer than IEEPB protocol by $64 \%$.

- $100 \%$ for SIEEPB- ACO is longer than the PEGASIS protocol by $56 \%$.

- $100 \%$ for SIEEPB- ACO is longer than IEEPB protocol by $14 \%$.

Furthermore, to compare our proposed protocol (SIEEPB- ACO) to other protocols that use ACO, taking ref. [12] as an 
example that been described previously in the literature survey section in this paper, the comparison will with his first scenario (PEG- ANT) for the network lifetime based on alive nodes for 100 nodes, $(100 m \times 100 m)$ WSN area, and initial energy 0.5. The comparison result shows that:

- For PEG- ANT protocol: FND (First Node Died) at 1\% enhanced by 50\% over PEGASIS protocol.

- For PEG- ANT protocol: LND (Last Node Died) at 100\% enhanced by 16\% over PEGASIS protocol.

- For SIEEPB- ACO protocol: FND (First Node Died) at 1\% enhanced by 133\% over PEGASIS protocol.

- For SIEEPB- ACO protocol: LND (Last Node Died) at 100\% enhanced by 56\% over PEGASIS protocol.

On the other hand, it is noticeable that PEGASIS protocol performs better than IEEPB protocol in $(30 \%, 50 \%$, and $70 \%)$ is due to small enhancement based on the suggested threshold and techniques that been described previously in this paper. IEEPB enhancement achieved by the author in ref. [9] were determined only by the start of the network lifetime and in the end when all nodes are dead but during the operation of data transmitting and receiving there is no enhancement and this is also presented in ref. [21] that compare PEGASIS- EEPB-IEEPB with his proposed protocol in the figure of network lifetime.

Result for scenario 2, where $E_{0}=1 J$ :

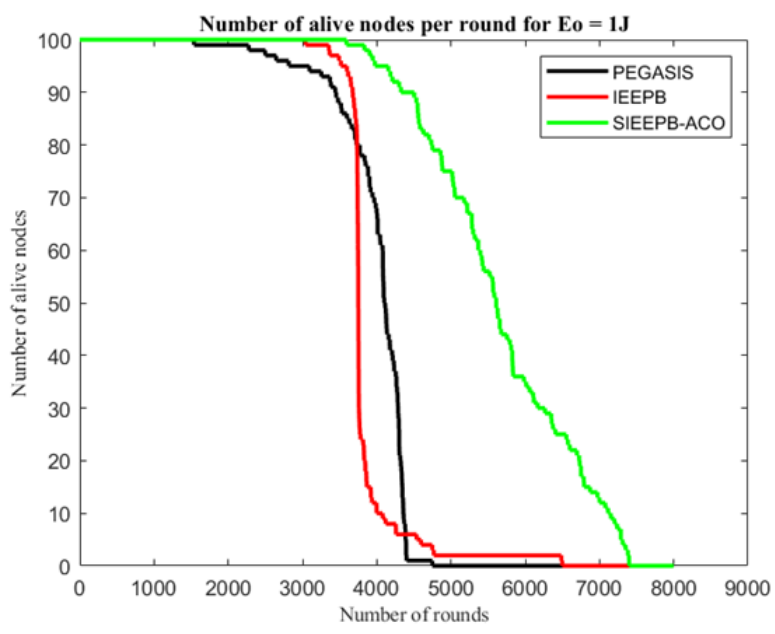

Figure 14: Number of alive nodes per round

TABLE V

NeTWORK LifETIME COMPARISION FOR $E_{o}=1 \mathrm{~J}$

\begin{tabular}{|c|c|c|c|}
\hline \multirow{2}{*}{ Nodes Death Rate } & \multicolumn{3}{|c|}{ Number of Rounds } \\
\cline { 2 - 4 } & PEGASIS & IEEPB & SIEEPB- ACO \\
\hline $1 \%$ & 1539 & 3069 & 3641 \\
\hline $10 \%$ & 3453 & 3678 & 4359 \\
\hline $30 \%$ & 3943 & 3743 & 5060 \\
\hline $50 \%$ & 4112 & 3752 & 5609 \\
\hline $70 \%$ & 4287 & 3760 & 6184 \\
\hline $100 \%$ & 4747 & 6507 & 7401 \\
\hline
\end{tabular}




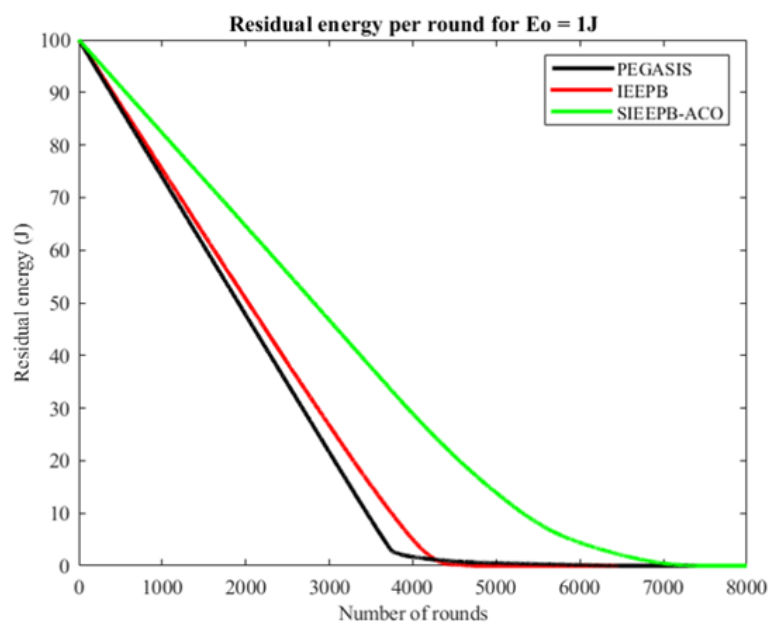

Figure 15: Residual average energy per round

In addition, normalized and residual energy of the network for SIEEPB- ACO in both scenarios showing a clear improvement for the behavior of network lifecycle.

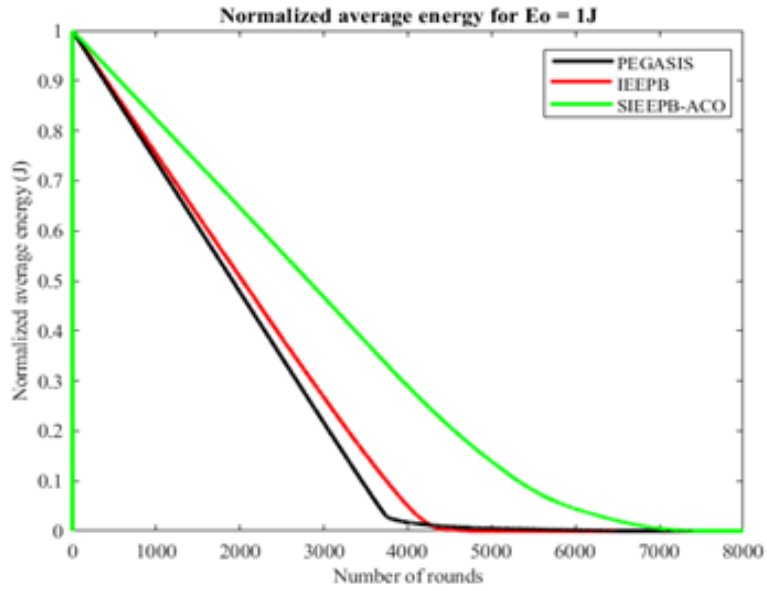

Figure 16: Normalized average energy per round

For the second scenario, when $E_{0}=1 J$. The number of rounds (see table V) when sensor nodes start to die at $1 \%$ is maximized due to the maximization of initial energy. For example, the number 1539 and 3069 in the first row (PEGASIS and IEEPB) means that the first dead node has been found after averagely 1539 and 3069 rounds when the original PEGASIS and it is improvement IEEPB protocol applied in the WSN, at same time for the proposed protocol (SIEEPBACO) the number of rounds where nodes start to die was bigger than the previous ones and this means that the lifetime of the WSN is extended in SIEEPB-ACO as shown in Fig. 14, Fig. 15 and Fig. 16. Same as when sensor nodes die at 10\% 
and all other percentages. In spite of this enhancement over PEGASIS and IEEPB, the increasing in initial energy will give a few more enhancement compare with the first scenario. Thus, to be clearer you can notice the prolonging of network lifetime when $(1 \%, 10 \%, 30 \%, 50 \%$, and 100\%) respectively of sensor nodes in the network have died (see Fig.17). So, the results of the enhancement show the average life span in:

- $1 \%$ for SIEEPB- ACO is longer than the PEGASIS protocol by $137 \%$.

- $1 \%$ for SIEEPB- ACO is longer than IEEPB protocol by $19 \%$.

- $10 \%$ for SIEEPB- ACO is longer than the PEGASIS protocol by $26 \%$.

- $10 \%$ for SIEEPB- ACO is longer than IEEPB protocol by $19 \%$.

- $30 \%$ for SIEEPB- ACO is longer than the PEGASIS protocol by $28 \%$.

- $30 \%$ for SIEEPB- ACO is longer than IEEPB protocol by $35 \%$.

- $50 \%$ for SIEEPB- ACO is longer than the PEGASIS protocol by $36 \%$.

- $50 \%$ for SIEEPB- ACO is longer than IEEPB protocol by $50 \%$.

- $70 \%$ for SIEEPB- ACO is longer than the PEGASIS protocol by $44 \%$.

- $70 \%$ for SIEEPB- ACO is longer than IEEPB protocol by $64 \%$.

- $100 \%$ for SIEEPB- ACO is longer than the PEGASIS protocol by $56 \%$.

- $100 \%$ for SIEEPB- ACO is longer than IEEPB protocol by $14 \%$.

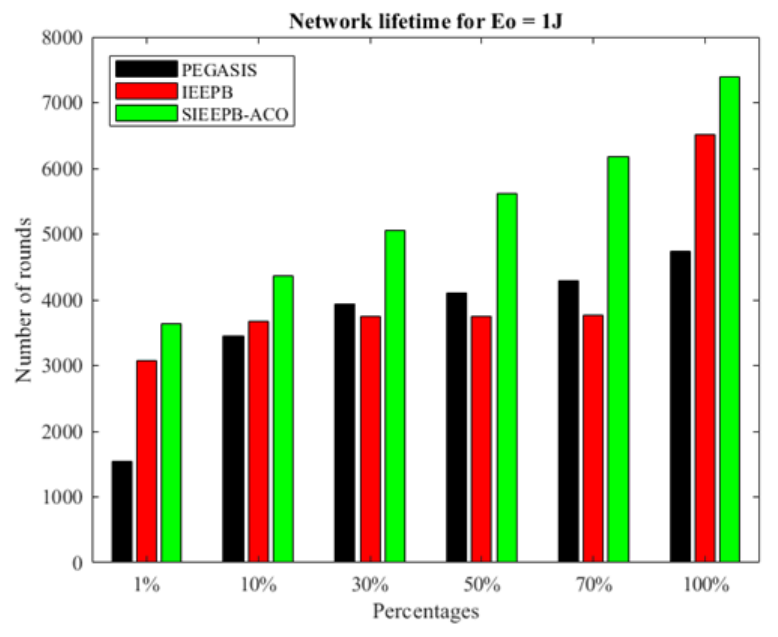

Figure 17: comparison of network lifetime

\section{Conclusions}

Due to the basic effective role that is played by WSN, it was necessary to go deeper and enhance more protocols belong to this kind of network Because of its great benefit in many fields. A hierarchal routing protocol gets all the attention in this work especially those who are specified in chain cluster-based routing protocols that been obvious in the second section: (literature survey). So in this paper, we were going through different chain based routing protocols and show the 
protocols that been improved over PEGASIS protocol as a core protocol for (EEPB, IEEPB). although, this paper proposes SIEEPB- ACO that is improved over IEEPB as a core protocol for two different scenarios based on initial energy by using one of the popular swarm intelligence optimization algorithms ACO. SIEEPB shows a high enhancement for the network performance and prolonging the network lifetime based on number of alive nodes per round by minimizing the energy consumption.

\section{Future Work}

Improved version SIEEPB- ACO is proposed for a single sink model in WSN but in future it can be implemented for multi-sink with a multi- chain model in WSN in which there will be multiple sink nodes and double chains in the same WSN area divided into two regions.

\section{REFERENCES}

[1] S. Misra and S. Goswami, " Network Routing: Fundamentals, Applications, and Emerging Technologies" , John Wiley \& Sons Ltd. 1st edition, Chapter 11, pp 285- 325, 2017.

[2] P. Dubey, Dr. S. Veenadhar, and Dr. S. Gupta, " Survey on Energy Efficient Clustering and Routing Protocols of Wireless Sensor Network " , International Journal of Scientific Research \& Engineering Trends, vol. 5, 1, pp. 212- 218, 2019.

[3] J.N. Al-Karaki, A.E. Kamal, " Routing techniques in wireless sensor networks: A survey ", IEEE Wireless Communication, vol. 11, pp. 6- $28,2004$.

[4] B. Bhushan, G. Sahoo, " Computational Intelligence in Sensor Networks" , vol. 776, Springer, chapter 10, pp. 215- 248, 2019.

[5] N. Sabor, S. Sasaki, M. Abo- Zahhad, and Sabah M. Ahmed, " A Comprehensive Survey on Hierarchical- Based Routing Protocols for Mobile Wireless Sensor Networks: Review, Taxonomy, and Future Directions", Wireless Communications and Mobile Computing, Hindawi, vol. 2017, pp. 1- 23, 2017.

[6] W. R. Heinzelman, A. Chandrakasan, and H. Balakrishnan, " Energy- Efficient Communication Protocol for Wireless Microsensor Networks" , In Proceedings of the 33rd Hawaii International Conference on System Sciences, Jan. 2000.

[7] S. Lindsey, C.S. Raghavendra, " PEGASIS: Power- efficient Gathering in Sensor Information Systems" , In Proceedings of IEEE Aerospace Conference, Big Sky, MT, USA, pp. 1125- 1130, March 2000.

[8] YU Yong- chang, WEI Gang, " An Improved PEGASIS Algorithm in Wireless Sensor Network" , Acta Electronica Sinica, vol. 36, pp. 1309- 1313, July 2008.

[9] F. Sen, Q. Bing, L. Tang, " An Improved Energy- Efficient PEGASIS- Based Protocol in Wireless Sensor Networks ", Eighth International Conference on Fuzzy Systems and Knowledge Discovery, 2011.

[10] Houang, R., Chen, Z. Xu, G., " Energy- aware routing algorithm in WSN using predication- mode " , IEEE, 2010 International Conference on Communications, Circuits, and Systems (ICCCAS), 2010.

[11] M. Raza Jafri, N. Javaid, A. Javaid, and Z. Ali Khan, " Maximizing the Lifetime of Multi- Chain PEGASIS Using Sink Mobility " World Applied Sciences Journal, vol 21, issue 9, pp. 1283- 1289, 2013.

[12] N. Ramluckun, and V. Bassoo, " Energy- efficient chain-cluster based intelligent routing technique for Wireless Sensor Networks " , Applied Computing and Informatics, 2018.

[13] B.G. Min, J. Park, H.G. Kim, and J.G. Shon, " Improvement of Multi- Chain PEGASIS Using Relative Distance " , In Park J. , Loia V. , Choo KK. , Yi G. (eds) Advanced Multimedia and Ubiquitous Engineering. MUE 2018, FutureTech 2018. Lecture Notes in Electrical Engineering, vol 518. Springer, Singapore, 2019.

[14] P. Kalkandha, C. Wahi, S. Sharma, and S. K. Sonbhadra " Current State of Art of Energy Efficient PEGASIS Routing Protocols in WSNs " , International Journal of Advanced Research in Computer Science and Software Engineering, Vol. 5, pp. 455- 460, 2015.

[15] S. Vhatkar, J. Rana, and M. Atique, " Performance Evaluation and QoS Analysis of EEPB and PDCH Routing Protocols in Wireless Sensor Networks " , IOSR Journal of Computer Engineering, vol. 17, pp. 101- 109, 2015.

[16] S. Sendra, L. Parra, J. Lloret, and S. Khan ", Systems and algorithms for wireless sensor networks based on animal and natural behavior ", International Journal of Distributed Sensor Networks, Vol. 11, issue. 3, pp.1- 19, 2015.

[17] A. A. Hussein and R.A. Khalid, " A Comparative Study of Swarm Intelligence- Based Optimization Algorithms in WSN ", Asian Journal of Engineering and Applied Technology, Vol. 8 No. 3, pp. 1- 7, 2019.

[18] R. Kumar, D. Kumar, " Hybrid swarm intelligence energy-efficient clustered routing algorithm for wireless sensor networks " , Journal of Sensors, Vol. 2016, pp. 1- 19, 2016.

[19] H. H. Mukhairez, A. Y. A. Maghari, " Performance comparison of simulated annealing, GA and ACO applied to TSP " , International Journal of Intelligent Computing Research (IJICR), Vol. 6, issue. 4, pp. 647- 654, 2015.

[20] R. Aggarwal, A. Mittal, R. Kaur, " Various optimization techniques used in wireless sensor networks " , International Research Journal of Engineering and Technology (IRJET), Vol. 3, issue. 6, pp. 2086- 2089, 2016.

[21] A. Jorio, E. S. Fkihi, B. Elbhiri, D. Aboutajdine, " An energy- efficient clustering routing algorithm based on geographic position and residual energy for wireless sensor network ", Journal of Computer Networks and Communications, vol. 2015, pp. 1- 11, 2015. 